\title{
Erratum to: Oxidative Stress and Adult Neurogenesis
}

\author{
Ti-Fei Yuan ${ }^{1} \cdot$ Simeng $\mathrm{Gu}^{1} \cdot$ Chunlei Shan ${ }^{2} \cdot$ Sergio Machado ${ }^{3,4} \cdot$ Oscar Arias-Carrión $^{5}$
}

Published online: 2 October 2015

(C) Springer Science+Business Media New York 2015

Erratum to: Stem Cell Rev and Rep (2015) 11:706-709

DOI 10.1007/s12015-015-9603-y

The correct author list for the article "Oxidative Stress and Adult Neurogenesis" should be:

Ti-Fei Yuan, Simeng Gu, Chunlei Shan, Sergio Machado, Oscar Arias-Carrión

In a previous version of the article, the last name for Dr. Machado was mis-spelled as Marchado.

The online version of the original article can be found at http://dx.doi.org/ 10.1007/s12015-015-9603-y

Ti-Fei Yuan

ytf0707@126.com

$\bowtie$ Chunlei Shan

shanclhappy@163.com

$\triangle$ Oscar Arias-Carrión

arias@ciencias.unam.mx

1 School of Psychology, Nanjing Normal University, Nanjing, China

2 Department of Rehabilitation Medicine, First Affiliated Hospital of Nanjing Medical University, Nanjing, China

3 Panic and Respiration, Institute of Psychiatry of Federal University of Rio de Janeiro, Rio de Janeiro, Brazil

4 Physical Activity Neuroscience, Physical Activity Sciences Postgraduate Program - Salgado de Oliveira University, Niterói, Brazil

5 Unidad de Trastornos del Movimiento y Sueño (TMS), Hospital General Dr. Manuel Gea González/IFC-UNAM, Mexico

City, Mexico 